\title{
Experiencias visuales y cognitivas a través de GIFS en Educación Primaria
}

Martín Caeiro Rodríguez - Universidad Internacional de La Rioja

Alberto Torres Pérez - Universidad Internacional de La Rioja

María del Mar Martínez Oña - Universidad Internacional de La Rioja
0000-0001-5616-3747

0000-0002-7991-1813

0000-0001-9231-7264

Recepción: 14.01.2019 | Aceptado: 21.01.2019

Correspondencia a través de ORCID: Martín Caeiro Rodríguez

0000-0001-5616-3747

Citar: Caeiro, M., Torres, A. y Martinez, MM. (2019). Experiencias visuales y cognitivas a través de GIFS en Educación Primaria. REIDOCREA, 8, 35-42. [ ]

\begin{abstract}
Resumen: EI GIF se ha convertido en un formato de expresión y comunicación habitual de las redes sociales, adquiriendo también protagonismo como un género artístico. Objetivos: El objetivo es generar experiencias cognitivas visuales en las que los niños sean protagonistas de su aprendizaje a través de ejemplos prácticos. Método: Para ello analizamos los archivos GIF clasificándolos a partir de tres criterios: intencionalidad, contexto y contenido, e identificando aplicaciones digitales (APPS) que permiten realizar GIFS. Asimismo, diseñamos una metodología bimodal que enlaza la identificación de APPS con la creación de GIFS. Resultados: Se han creado nueve GIFS a partir de contenidos curriculares artísticos utilizando APPS y procesos adecuados a la etapa de Primaria. Discusión: El análisis de APPS y los GIFS creados indican que ambos elementos asociados permiten trabajar contenidos en Educación Primaria presentando la información de un modo más dinámico y visual.
\end{abstract}

Palabras clave: Imagen Dinámica | Cognición

\section{Visual and cognitive experiences through GIFS in Primary Education}

\begin{abstract}
The GIF has become a habitual form of expression and communication in the social media, also acquiring importance as an artistic genre. Objectives: The aim is to generate visual cognitive experiences in which children are the protagonists of their learning through practical examples. Method: To this end, we analyse GIF files by classifying them according to three criteria: intentionality, context and content, and identifying digital applications (APPS) that allow GIFS to be made. We also designed a bimodal methodology that links the identification of APPS with the creation of GIFS. Results: Nine GIFS were created based on artistic curricular content using APPS and processes appropriate to the Primary level. Discussion: The analysis of APPS and the GIFS created indicate that both associated elements allow work on content in Primary Education presenting the information in a more dynamic and visual way.
\end{abstract}

Keywords: Dynamic Image | Cognition

\section{Introducción. La captación del movimiento como aprendizaje. Conceptos asociados al movimiento y algunos inventos}

Durante los siglos XVIII y XIX comenzaron a desarrollarse y perfeccionarse diversos juguetes ópticos, herederos de la Linterna Mágica, la Cámara Oscura y del Teatro de Sombras, considerados hoy como los primeros inventos precursores de la captación del movimiento. A estos inventos los acompañaban teorías acerca de cómo percibimos el movimiento. Peter Mark Roget en 1824 ofreció la primera explicación científica y técnica, en la que se estudió a fondo el fenómeno de la percepción del movimiento. Otro concepto asociado históricamente al movimiento es el de la persistencia de la visión o "persistencia retiniana" establecido por Joseph-Antoine Ferdinand Plateau en la que se refiere a una característica de nuestros ojos que hace que en el cerebro queden guardadas por un instante las imágenes percibidas. Esta idea tuvo una gran repercusión en la época, dando lugar a diferentes inventos. Plateau inventó un primitivo dispositivo estroboscópico, el Fenaquistiscopio (Figura 1), que era capaz de proporcionar la ilusión de una imagen en movimiento a partir de una secuencia de imágenes fijas. A este invento le sucederían otros como el Zoótropo, que derivó en el 
Praxinoscopio de Reynaud y su variable, el Teatro-Óptico. En 1892 se proyectaron en París las "Patomimes Lumineuses" de Reynaud, a partir de la proyección de dibujos móviles considerados como el primer espectáculo de animación (Figura 2). También debemos destacar por su sencillez de elaboración el Taumátropo y el Folioscopio, todos inventos basados en la representación de una secuencia que se repite en bucle. Esta progresión inventiva culminaría con la invención del cinematógrafo por los Hermanos Lumière en 1895. (Poyet, 1982; Emblen, 1970)
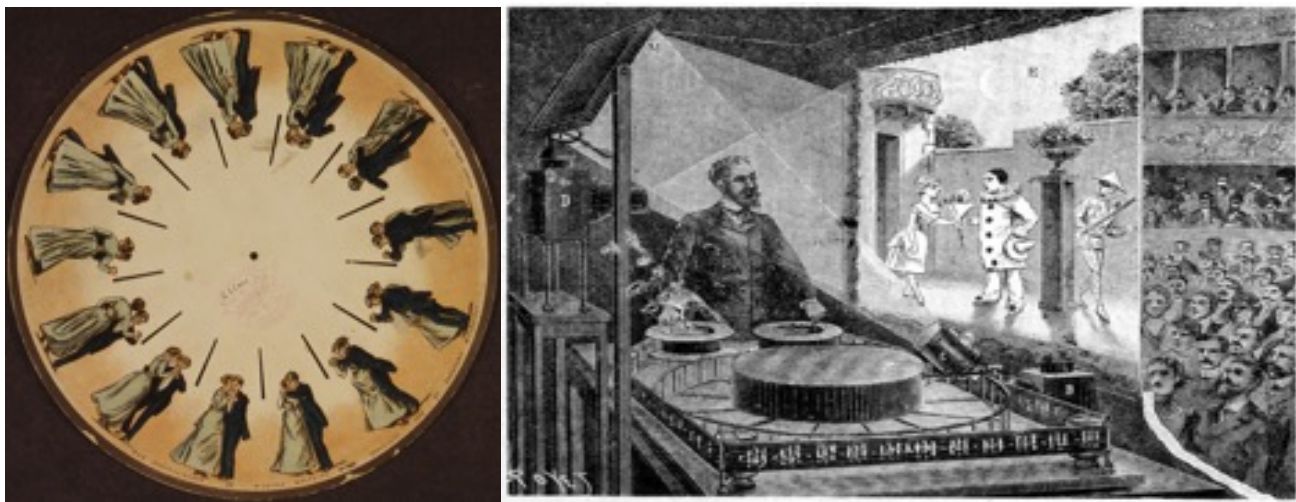

Figuras 1 y 2 :

1. Izquierda: Fenaquistiscopio, Joseph Plateau (1895)

Fuente: https://commons. wikimedia.org/w/index.php?curid=1853472

Para ver la animación GIF: https://es.wikipedia.org/wiki/Fenaquistoscopio\#/media/File:Phenakistoscope_3g07690b.gif 2. Derecha: Reynaud y su Teatro Óptico (1892).

Fuente: https://commons. wikimedia.org/w/index.php?curid=1441071

El cine primero y la televisión después (Gubern, 1995, 2007) y el desarrollo del pensamiento y el lenguaje visual (Barthes, 1986, Arnheim, 1969, 1981, Villafañe, 2006) hicieron que la experiencia de la imagen en movimiento se extendiese masivamente a lo largo del siglo XX. Hoy, con los dispositivos digitales móviles, presentes en la cotidianidad familiar, educativa y social, podemos considerar que desde pequeños vamos educándonos en un pensamiento caracterizado por una imagen dinámica. Las tecnologías digitales (Saura, 2011) nos permiten a los docentes actualmente pasar de la representación gráfica estática a la representación dinámica (Urraca, 2015) y trabajar en el aula con niños y niñas de un modo más lúdico, artístico y visual los conceptos y contenidos presentes en el currículo (Eisner, 2012), contribuyendo al desarrollo cognitivo de su pensamiento y adquisición de lenguajes (Bruner, 2002). Los GIFS, dada la sencillez que presentan a la hora de crearlos, resultan ideales para introducir a los alumnos desde las primeras etapas educativas en la comprensión de contenidos diversos al mismo tiempo que se trabaja desde la concepción de una imagen más dinámica esos contenidos curriculares.

\section{Los GIFS y el conocimiento en bucle}

Programas y aplicaciones cada vez más sencillos permiten en la actualidad que los niños experimenten con sus propias creaciones animadas. En el caso del GIF son suficientes 3 imágenes para generar la ilusión del movimiento. Podemos considerar el GIF como un género expresivo propio encontrando espacios virtuales dedicados exclusivamente a este medio, tales como GIF ART, GIF OF ARTS o incluso festivales temáticos como GIF Arts Festival de Los Ángeles o Festival International du GIF. Hoy, con los dispositivos digitales móviles, los docentes podemos pasar de la representación gráfica estática (Urraca, 2015) a la representación dinámica del contenido.

Los GIFS, dada su sencillez a la hora de crearlos, resultan ideales para introducir a los alumnos desde las primeras etapas educativas en la comprensión de contenidos 
diversos. En Internet podemos encontrar numerosos ejemplos de creaciones GIF. Conviene destacar que muchos tienen un carácter puramente lúdico, creados desde una voluntad humorística y mediática, otros artística y otros cognitiva. A nosotros nos han interesado aquellos GIFS creados para transmitir conocimiento y que aportan una experiencia estética al mismo tiempo que comportan una calidad mínima artística en su elaboración y diseño.

\section{Objetivos}

El objetivo general de este trabajo es posibilitar experiencias cognitivas visuales en las que los niños sean protagonistas de su aprendizaje ofreciendo a los maestros ejemplos prácticos y en los que pasamos de las Tecnologías de la Información y la Comunicación a las Tecnologías para el Aprendizaje y el Conocimiento (Roser, 2011). Para ello, como acciones específicas, identificamos APPS que permiten generar GIFS, y analizamos y categorizamos los tipos que existen estableciendo la hipótesis de que estos son recursos adecuados para la compresión de contenidos en la etapa de Primaria. Se trata de aportar a los docentes un marco de referencia para sus actividades y que dispongan de ejemplos prácticos que les guíen acerca de los procesos de creación de un GIF y de cuáles son las APPS más adecuadas a cada edad y contenido. En este sentido, no utilizamos el GIF en una vertiente de entretenimiento o puramente artística, sino como vehículo de conocimiento para el alumno y como recurso didáctico para el docente.

\section{Método}

Se ha seguido una metodología de investigación-acción y realizado una evaluación comprensiva (Stake, 2006), tanto de las APPS como de los GIFS a partir de un conocimiento experiencial y personal de los procesos de creación de imágenes dinámicas. La evaluación comprensiva significa guiarse por la experiencia y basarse en gran medida en la interpretación personal. Su rasgo esencial es la comprensión (receptividad o sensibilidad) de cuestiones o problemas clave en relación con los intereses de la investigación-acción (Stake, 2006, citado por Rico, 2017). En el caso que se plantea en este trabajo, el contexto es la Educación Artística en Primaria y unos contenidos curriculares que se exploran, experimentan e investigan desde la creación (Caeiro, 2018) en formato GIF por medio de APPS.

Para el estudio de los GIF y la búsqueda y selección de APPS hemos partido del modelo diseñado por Rico (2017) quien se inspira a su vez en el modelo ideado por el (Observatorio de Educación Patrimonial de España (OEPP) para investigaciones que usan Internet como fuente de búsqueda y parten de estándares de evaluación: 1) Localizar: Determinación de estándares de calidad, Localización de APPS a través de buscadores de internet; 2) Inventariar: Exploración de APPS educativas; 3) Clasificar: Selección, discriminación, Diseño de una ficha para establecer criterios de inclusión y exclusión; 4) Analizar: Exploración de APPS; 5) Sistematizar: Diseño de una herramienta de evaluación de APPS; 6): Evaluar: Evaluación de APPS basada en criterios y estándares, Evaluación contextual y comprensiva en el aula; 7) Estandarizar: Recogida de datos, análisis y resultados, Análisis estadístico-descriptivo. Este método lo hemos adaptado a nuestra investigación marcando unas acciones según los GIF y otras según las APPS. (Tabla 1).

Para la elección del contenido a trabajar en formato GIF y para crearlos, se ha partido de los Bloques de contenidos (Tabla 2) que marca la legislación (BOE, 2014), situando en ellos los escogidos por cada uno de los autores del artículo. 
Para el análisis y selección de las APPS hemos tenido en cuenta la dimensión de Usabilidad establecida por Rico (2017: 365). En este sentido, nos hemos centrado en algunos de los criterios establecidos por la autora asociados a la usabilidad: 1) Acceso; 2) Memorabilidad; 3) Accesibilidad, 4) Seguridad, 5) Contexto, 6) Interfaz.

Finalmente, para el análisis y clasificación de GIFS hemos seguido tres criterios: 1) la intencionalidad del autor: hacer humor, hacer arte, hacer conocimiento, combinación de arte y conocimiento, 2) el contexto en el que se difunde: espacio de entretenimiento, página especializada; 3 ) el contenido: humorístico, artístico, cognitivo, combinación de lo artístico y lo cognitivo.

\begin{tabular}{|c|c|c|c|}
\hline \multicolumn{4}{|c|}{$\begin{array}{l}\text { Tabla 1. Acciones metodológicas bimodales según los GIFS y según } \\
\text { ACCIONES DE LA INVESTIGACIÓN }\end{array}$} \\
\hline \multicolumn{2}{|c|}{ EN RELACIÓN A LOS GIFS } & \multicolumn{2}{|c|}{ EN RELACIÓN A LAS APPS } \\
\hline Localizar & $\begin{array}{l}\text {-Determinación de estándares de calidad } \\
\text {-Localización de GIFS a través de } \\
\text { buscadores de internet tanto en } \\
\text { ordenador, como en Tabletas y } \\
\text { Smartphone }\end{array}$ & Localizar & $\begin{array}{l}\text {-Determinación de estándares de } \\
\text { calidad } \\
\text {-Localización de Apps a través de } \\
\text { buscadores de internet, tanto en } \\
\text { ordenador, como en Tabletas y } \\
\text { Smartphone }\end{array}$ \\
\hline Inventariar & Almacenamiento de GIFS en una base & Inventariar & $\begin{array}{l}\text { Almacenamiento de Apps que permiten } \\
\text { crear GIFS }\end{array}$ \\
\hline Clasificar & $\begin{array}{l}\text {-Selección de GIFS } \\
\text {-Identificación según tipología: } \\
\text { humorísticos, artísticos, cognitivos en base } \\
\text { a tres criterios: intencionalidad, contexto, } \\
\text { contenido }\end{array}$ & Clasificar & $\begin{array}{l}\text {-Selección, discriminación de Apps } \\
\text { para Tableta y Smartphone } \\
\text {-Diseño de una ficha para establecer } \\
\text { criterios de inclusión y exclusión en } \\
\text { Primaria }\end{array}$ \\
\hline Analizar & Exploración de GiFS cognitivos & Analizar & $\begin{array}{l}\text { Exploración de Apps que permiten } \\
\text { crear GIFS en Tableta y Smartphone }\end{array}$ \\
\hline Asociar & $\begin{array}{l}\text { Identificación de contenidos de Primaria } \\
\text { válidos para crear GIFS }\end{array}$ & Asociar & $\begin{array}{l}\text { Identificación de las herramientas que } \\
\text { contiene cada APP para la creación de } \\
\text { un GIF }\end{array}$ \\
\hline CREAR & Creación de GIFS cognitivos & DESCARTAR & $\begin{array}{l}\text { Identificación de las APPS que no se } \\
\text { adaptan a las necesidades de Primaria }\end{array}$ \\
\hline \multicolumn{4}{|c|}{$\begin{array}{l}\text { Sistematizar: Identificación de los procesos necesarios a la creación de un contenido en formato GIF a partir de una APP } \\
\text { Estandarizar: Los procesos que permiten crear GIFS adaptados a cada curso de Primaria } \\
\text { Evaluar: Evaluación comprensiva de los GIFS creados por el investigador }\end{array}$} \\
\hline
\end{tabular}

Tabla 2. Bloques de contenidos artísticos en Educación Primaria Bloques de contenidos relacionados con expresión plástica y visual en Educación Primaria

\begin{tabular}{|cccc|c|c|}
\hline Bloque 1 Educación Audiovisual & $\begin{array}{l}\text { Está referido al estudio de la imagen en todas sus manifestaciones, tanto } \\
\text { visual como audiovisual, en el que cobran una gran relevancia las } \\
\text { aportaciones que las Tecnologías de la Información y la Comunicación } \\
\text { realizan al mundo de la imagen. }\end{array}$ \\
\hline Bloque 2 & Expresión Plástica & $\begin{array}{l}\text { Hace referencia al conjunto de conceptos y procedimientos que } \\
\text { tradicionalmente han estado asociados al área. Experimenta con materiales } \\
\text { y técnicas diversas en el aprendizaje del proceso de creación. }\end{array}$ \\
\hline Bloque 3 & Dibujo Geométrico & $\begin{array}{l}\text { Incluye el desarrollo desde el punto de vista gráfico de los saberes } \\
\text { adquiridos desde el área de matemáticas en el apartado de geometría. }\end{array}$ \\
\hline
\end{tabular}

\section{Instrumentos}

Los dispositivos con los que hemos trabajado para identificar las APPS y realizar los GIF han sido Internet, nuestros Smartphones, ordenadores y Tabletas. Teniendo en cuenta que la mayoría de las Aplicaciones se basan en la existencia de una base de 
archivos ya sean gráficos, imágenes, fotografías... en ocasiones es necesario realizar previamente con otros procedimientos e instrumentos las imágenes que posteriormente subiremos a la aplicación para crear el GIF. Y dependiendo del contenido estas imágenes que compondrán el GIF serán gráficas, fotográficas, pictóricas, físicas o digitales. En nuestro caso, hemos utilizado tanto recursos y herramientas de creación grafico-plástica como digitales, según las posibilidades y limitaciones de la APP.

Los programas de edición digitales como Adobe Photoshop, Adobe Illustrator, Adobe Animate, Corel Draw o Gimp, nos permiten realizar GIFS animados. Sin embargo, las aplicaciones digitales (APPS) generan posibilidades más rápidas y de fácil uso para la creación de un GIF permitiendo que los propios alumnos los creen con el smartphone o la tableta digital y sin tener que utilizar programas complejos.

\section{Procedimiento}

A partir del diseño de nuestra metodología bimodal hemos llevado a cabo una revisión en Internet identificado diversos programas y APPS que permiten hacer animaciones GIF. Los criterios generales aplicados en la búsqueda de GIFS fueron:

- Página más visitada según el buscador de GOOGLE Chrome al poner GIF; GIFS, GIF de Arte.

- Diferenciar páginas de entretenimiento dedicadas a los GIF de páginas que fuesen profesionales o educativas.

- Identificar GIFS de carácter cognitivo y artístico y otros de carácter comunicativo.

- Encontrar GIFS en los que la animación guardase relación con contenidos curriculares de la Etapa de Primaria.

- Identificar GIFS sencillos, pero con calidad e interés cognitivo y artístico.

- Que la dimensión estética y el diseño gráfico formasen parte de la animación GIF y no fuesen una ocurrencia memética (un meme).

- Aproximarnos a la tipología de las aplicaciones ideadas para la creación de GIFS.

- Identificar las APPS más apropiadas según edad y usabilidad.

Una vez terminada la búsqueda online de APPS y decidido el contenido que cada autor trabajaría, se procedió a experimentar con diferentes APPS para identificar la más adecuada al contenido a animar, y a crearlos. El proceso de creación de cada GIF ha sido similar en cada autor, con variables sobre el inicio:

- GIFS 1 y 2: Bocetos previos a lápiz, bolígrafo sobre papel, uso de colores... para ver las posibilidades visuales del contenido. GIF 3: Creación fotograma a fotograma en Drawisland. GIF 4 y 6: Creación directa con el programa de edición GIMP por capas. GIF 5: Creación directa con el programa Corel Draw sobre una base predefinida de capas exportando fotograma a fotograma cada instante de la creación en formato JPG. GIF 7 y 8: Búsqueda de fotos en internet y manipulación con el programa Adobe llustrator creando capas. GIF 9: creación directa usando los dedos en FlipaClip.

- Captación fotográfica, escaneado o exportado durante la fase de creación pensando en las que compondrán el GIF. 
- Carga de las fotografías o frames realizados en la fase de creación al banco de imágenes del Smartphone, la Tableta o el Ordenador.

- Paso a los recursos APP: experimentación con las posibilidades de las diferentes herramientas que ofrece: formato, colores, texto... y carga de las fotografías o frames.

- Elaboración final y generación de la animación GIF, ajuste de los frames por segundo.

- Subir el archivo a Google Drive generando enlace para compartir.

\section{Resultados}

En relación a la búsqueda de APPS (Tabla 3) se utilizó el espacio propio de Android e IOS, descargando y experimentando con cada APP en nuestros propios dispositivos. La búsqueda se llevó a cabo de forma individual por cada uno de los investigadores, procediendo después a una puesta en común para establecer la idoneidad y usabilidad de cada APP según la edad de los niños, así como la adecuación de cada contenido escogido para generar el GIF. Algunas APPS analizadas ofrecen publicidad constante, otras limitan las funciones a la versión PRO de pago y por ello sólo algunas son aptas para introducir en Primaria. Desde ahí hemos ido marcando según su complejidad o facilidad de uso un valor de 0 (máxima facilidad) o 10 (máxima complejidad). Asimismo, se ha valorado la edad aproximada que mejor encaja con esa APP. Aunque, evidentemente, esto dependerá también del contenido que se vaya a trabajar. Del análisis de los diferentes GIF hemos identificado unos de carácter meramente humorístico de otros fundamentalmente artísticos y otros de carácter cognitivo, y algunos ejemplos donde lo cognitivo y lo artístico se entrelazan. Los que nos han interesado y recomendamos para trabajar en el aula de Primaria son los que articulan 1) la intencionalidad del autor con la combinación de arte y conocimiento, 2) el contexto que ofrece al mismo tiempo entretenimiento y especialización; 3) el contenido que es artístico y cognitivo.

\begin{tabular}{|c|c|c|c|}
\hline \multicolumn{4}{|c|}{ Tabla 3. APPS y entornos online para crear GIFS y análisis según su usabilidad } \\
\hline \multicolumn{4}{|c|}{ ALGUNAS APPS Y ENTORNOS ONLINE PARA GENERAR ANIMACIONES GIFS } \\
\hline APP/ESPACIO & DESCRIPCIÓN & Usabilidad & Edad aconsejada \\
\hline GIF OF ARTS & $\begin{array}{l}\text { Es una aplicación para Smartphone y Tableta que sirve como espacio } \\
\text { divulgativo de GIFS. }\end{array}$ & 3 & 6 \\
\hline Draw Island: & $\begin{array}{l}\text { Es una herramienta en línea gratis para la creación de dibujos y } \\
\text { animaciones GIF simples. Tiene una paleta de herramientas amplia. } \\
\text { Permite descargar los cuadros y a partir de ellos generar el GIF. }\end{array}$ & 5 & 7 \\
\hline GIF Maker & $\begin{array}{l}\text { Es gratuita y no genera publicidad Se genera un GIF que se puede } \\
\text { enviar a la red para publicar o descargar online. No permite modificar la } \\
\text { velocidad. }\end{array}$ & 7 & 6 a 11 \\
\hline GIF Maker & $\begin{array}{l}\text { Es gratuita y no genera publicidad. Se genera un GIF que se puede } \\
\text { enviar a la red para publicar o descargar online. No permite modificar la } \\
\text { velocidad. }\end{array}$ & 7 & 6 a 11 \\
\hline 5s GIF & $\begin{array}{l}\text { Permite utilizar de forma gratuita diversas herramientas, filtros, velocidad, } \\
\text { añadir texto... }\end{array}$ & 9 & 8 a 11 \\
\hline GF Factory & $\begin{array}{l}\text { Permite crear rápidamente GIFS. Y ofrece herramientas para modificar, } \\
\text { por ejemplo, la velocidad de reproducción. Algo muy útil. Pero no permite } \\
\text { ni compartir ni descargar el GIF. }\end{array}$ & 8 & 10 y 11 \\
\hline GIF Maker & $\begin{array}{l}\text { Sin la versión Pro de Pago no permite trabajar con más de } 6 \text { GIFS. } \\
\text { Permite uso de filtros. }\end{array}$ & & \\
\hline Gif Pro & $\begin{array}{l}\text { De forma fácil y rápida permite crear GIF animados, videos, etcétera que } \\
\text { se pueden compartir en redes sociales. Presenta publicidad para adultos. }\end{array}$ & 8 & 8 \\
\hline GIFSHOP & Se trata de una aplicación fácil de usar, aunque incluye publicidad. & 3 & 8 \\
\hline Gif Creator & Sin la versión Pro de pago no permite trabajar con más de 3 GIFS. & & \\
\hline Glf Creator & Se cierra durante el proceso. & & \\
\hline FlipaClip & $\begin{array}{l}\text { Se trata de una aplicación fácil de usar que también permite crear la } \\
\text { animación dibujando sobre la tableta o Smartphone fotograma a } \\
\text { fotograma. }\end{array}$ & 6 & 10 y 11 \\
\hline
\end{tabular}


Los GIFS creados por los autores (Tabla 4) son reproducibles, tanto por los maestros como por los niños al haber seguido los autores procesos asequibles a las edades de los niños que cursan Primaria y teniendo en cuenta los conocimientos adquiridos por los maestros en el Grado en materia artística. Se debe tener en cuenta la orientación a la edad, tanto de la APP como del GIF cognitivo, ya que hay APPS con publicidad o poco intuitivas y GIFS cuyo contenido requiere de un pensamiento y razonamiento más abstracto.

Tabla 4. Ejemplos creados por los autores teniendo en cuenta su adecuación al contenido y a la edad

\begin{tabular}{|c|c|c|c|c|}
\hline Contenido & Descripción & APP & Edad & GIF \\
\hline Geometría & $\begin{array}{l}\text { Este GIF permite comprender mejor el paso de una a dos } \\
\text { y a tres dimensiones espaciales. }\end{array}$ & $5 \mathrm{~s} \mathrm{GIF}$ & 8 & \\
\hline Educación Visual & $\begin{array}{l}\text { Este GIF permite trabajar las Figuras Retóricas Visuales } \\
\text { y facilitar su comprensión. }\end{array}$ & $5 \mathrm{~s}$ GIF & 7 & \\
\hline $\begin{array}{l}\text { Educación Visual } \\
\text { y Geometría }\end{array}$ & $\begin{array}{l}\text { Este GIF permite trabajar la ilusión de la deformación de } \\
\text { un objeto y la trayectoria según el ángulo. }\end{array}$ & $5 \mathrm{~s}$ GIF & 7 & \\
\hline Expresión Plástica & $\begin{array}{l}\text { Este GIF permite comprender la generación de colores } \\
\text { secundarios a partir de los primarios. }\end{array}$ & GIF Maker & 7 & \\
\hline Geometría & $\begin{array}{l}\text { Este GIF permite comprender el proceso de dibujo } \\
\text { técnico de un pentágono inscrito en una circunferencia. }\end{array}$ & GIF Maker & 11 & \\
\hline Expresión Plástica & $\begin{array}{l}\text { Este GIF permite trabajar la elaboración de un boceto, } \\
\text { pasando de la línea y el encaje al volumen. }\end{array}$ & GIF Maker & 10 & \\
\hline $\begin{array}{l}\text { Educación Visual } \\
\text { y Expresión Plástica }\end{array}$ & $\begin{array}{l}\text { Este GIF permite trabajar la comprensión del movimiento } \\
\text { y la expresión plástica del color. }\end{array}$ & GIFSHOP & 8 & \\
\hline $\begin{array}{l}\text { Educación Visual } \\
\text { y Expresión Plástica }\end{array}$ & $\begin{array}{l}\text { Este GIF permite trabajar la animación a partir de la } \\
\text { línea. }\end{array}$ & GIFSHOP & 8 & \\
\hline $\begin{array}{l}\text { Educación Visual } \\
\text { y Expresión Plástica }\end{array}$ & $\begin{array}{l}\text { Este GIF permite trabajar el paso de lo abstracto } \\
\text { (mancha) a lo concreto (estrella) así como con la } \\
\text { expresividad de los colores complementarios. }\end{array}$ & FlipaClip & 6 & \\
\hline
\end{tabular}

\section{Discusión}

De la experimentación llevada a cabo con las APPS a partir de contenidos curriculares de Primaria, hemos comprobado que es viable, posible y deseable que los maestros incorporen el GIF como recurso para el desarrollo cognitivo de los niños, y como apoyo a otras experiencias donde la información pueda ser estática. En este sentido, podemos afirmar que el GIF es un recurso adecuado para la compresión de contenidos en la etapa de Primaria. Hemos identificado que hay diversas posibilidades con las que realizar experiencias animadas en las aulas en las que los niños pueden ser protagonistas de su aprendizaje implicándose activamente desde la generación de los GIFS. Aunque el mundo de las APPS resulta hoy en día abrumador, las posibilidades que nos ofrecen a los docentes para incorporar experiencias contemporáneas en el aula, son numerosas (Escaño, 2010; Briggs y Blythe, 2013; Saura, 2011). EI GIF es un medio útil y ofrece un formato idóneo para trabajar con él en el aula y como recurso didáctico, ya que permite trabajar cognitivamente la información de un modo más dinámico y visual, enlazando además procesos físicos y analógicos con otros digitales (Gómez y Sáez, 2012; Marín, 2011) siguiendo modelos más contemporáneos como el b-learning (Bajardi, 2015), en la idea de enseñar y aprender en una "aula sin muros" (McLuhan, 1974). Las generaciones actuales, inmersas en una era mediática, han desarrollado un pensamiento más cinematográfico de la información. No obstante, la mayoría de los contenidos siguen trabajándose en el aula y en las diferentes materias de forma estática o literal. Por otro lado, teniendo en cuenta el uso que las redes sociales e internet le da a los GIFS, dominando sin lugar a dudas el GIF memético o humorístico, se hace necesario que el educador incorpore en este formato, experiencias que permitan ver a los niños que hay un uso y aplicación del GIF más interesante desde el punto de vista cognitivo y artístico. En relación a la 
prospectiva, queda por llevar a cabo la fase en las aulas de Primaria por maestros en activo para poder validar los instrumentos y profundizar en las categorías, criterios y dimensiones por ahora consideradas, avanzando con el uso de otros modelos (Hernández, 2012, Kortabitarte et al., 2017). Esperamos que este estudio inicial que enlaza investigación y creación sirva para incentivar su incorporación al aula en el contexto de la educación artística.

\section{Referencias}

Arnheim, R. (1969). El pensamiento visual. Barcelona: Paidós Ibérica.

Arnheim, R. (1981). Arte y Percepción Visual. Psicología del ojo creador. Madrid: Alianza Editorial.

Bajardi, A. (2015). B-Learning y arte contemporáneo en educación artística: Construyendo identidades personales $y$ profesionales. Tesis doctoral: Universidad de Granada.

Barthes, R. (1986). Retórica de la imagen. Lo obvio y lo obtuso. Madrid: Paidós Ibérica.

BOE (2014). Real Decreto 126/2014, de 28 de febrero, por el que se establece el currículo básico de la Educación Primaria.

Briggs, J., \& Blythe, M. (2013, August). Apps for art's sake: resistance and innovation. In Proceedings of the 15th international conference on Human-computer interaction with mobile devices and services, 45-54.

Bruner, J. (2002). El desarrollo de los procesos de representación (Capítulo 5). En JL. Linaza: Acción, pensamiento y lenguaje. Madrid: Alianza Editorial.

Caeiro, M. (2018). Aprendizaje Basado en la Creación y Educación Artística: proyectos de aula entre la metacognición y la metaemoción. Arte, Individuo y Sociedad, 30(1), 159-177.

Eisner, WE. (2012). El arte y la creación de la mente: el papel de las artes visuales en la transformación de la conciencia. Barcelona: Paidós Ibérica.

Emblen DL. (1970). Peter Mark Roget: the word and the man. Londres: Longman.

Escaño, C. (2010). Hacia una educación artística 4.0. En Arte, Individuo y Sociedad, 22(1), 135-144.

Gómez, RA. y Sáez, CM. (2012). La web 2.0 como herramienta didáctica de apoyo en el proceso de enseñanza aprendizaje: aplicación del blog en los estudios de Bellas Artes. Tesis doctoral: Universidad Complutense de Madrid.

Gubern, R. (1995). Historia del cine. Barcelona: Editorial Lumen.

Gubern, R. (2007). Del bisonte a la realidad virtual: la escena y el laberinto. Barcelona: Anagrama.

Hernández, J. (2012) Instrumento para la valoración preliminar del uso de Apps en Educación Primaria, en II Congreso Internacional de Investigación e Innovación en Educación Infantil y Educación Primaria. Universidad de Murcia.
Kortabitarte, A., Ibáñez-Etxeberria, A., Luna, U., Vicent, N., Gillate, I., Molero, B. y Kintana, J. (2017). Dimensiones para la evaluación de aprendizajes en APPs sobre patrimonio. Pulso. Revista de educación, 40, 17-33.

Marín, T. (2011). Experiencia de innovación docente GOUMH: aprendizaje colaborativo en Bellas Artes con APPS de Google (Actas), en Congreso Internacional de Innovación docente. Universidad de Cartagena: Cartagena.

McLuhan, M. (1974). El aula sin muros. Barcelona: Laia.

Poyet, LE. (1892) First public performance of Reynaud's 'Theatre Optique' in Paris. (Archived by WebCite $\AA$ at http://www.webcitation.org/75RiYzsEe)

Rico, AB. (2017). Evaluación del uso de las APPs que abordan los procesos creativos en la educación artística formal. Tesis Doctoral: Universidad de Valladolid.

Roser, L. (2011). De las TIC a las TAC: tecnologías del aprendizaje y del conocimiento. Anuario ThinkEPI, 5, 45-47.

Saura, A. (2011). Innovación educativa con TIC en Educación Artística, Plástica y Visual. Líneas de investigación y estudios de casos. Madrid: Mad S.L.

Stake, RE. (2006). Evaluación comprensiva y evaluación basada en estándares. Barcelona: Ariel.

Urraca, ML. (2015). Representación del movimiento en el dibujo: 5-8 años. Tesis doctoral: Universidad de la Rioja.

Villafañe, J. (2006). Introducción a la teoría de la imagen. Madrid: Pirámide. 